\title{
Análise do Desempenho de Estudantes com base em Perfis de Interação
}

\author{
Hiran N. M. Ferreira ${ }^{1}$, Fabiano A. Dorça ${ }^{2}$, Renan G. Cattelan ${ }^{2}$ \\ ${ }^{1}$ Instituto Federal de Educação, Ciência e Tecnologia do Sul de Minas Gerais \\ (IFSULDEMINAS) - Passos/MG, Brasil \\ ${ }^{2}$ Faculdade de Computação - Universidade Federal de Uberlândia (UFU) \\ Uberlândia/MG, Brasil
}

\begin{abstract}
Computational resources can be used to support educational activities, both in face-to-face teaching and in distance learning, creating more interactive environments. This work investigates the effectiveness of the use of Adaptive and Intelligent Education Systems with ubiquitous computing resources. The objective is to verify whether the interaction profile of the student in the platform influences its performance. In the experiments, six classes were followed during three academic semesters, comprising a total of 119 students. A clustering algorithm has been used to help define different groups from the perspective of how they use the environment. The results show that the interaction profile has a direct (statistically proven) influence on student performance.
\end{abstract}

Resumo. Recursos computacionais podem ser empregados para apoiar atividades educacionais, tanto no ensino presencial quanto no ensino a distância, criando ambientes mais interativos. Este trabalho investiga a efetividade da utilização de Sistemas Adaptativos e Inteligentes para Educação com recursos ubíquos de computação. O objetivo é verificar se o perfil de interação do estudante na plataforma influencia seu desempenho. Nos experimentos, foram acompanhadas seis turmas durante três semestres letivos, englobando um total de 119 estudantes. Um algoritmo de agrupamento foi utilizado para ajudar a definir diferentes grupos sob a perspectiva de como utilizam o ambiente. Os resultados mostram que o perfil de interação tem influência direta (comprovada estatisticamente) no desempenho do estudante.

\section{Introdução}

A integração de recursos computacionais a ambientes educacionais é uma tendência cada vez mais forte. Tais ambientes são equipados com ferramentas que podem promover uma melhora significativa na aprendizagem e no processo de construção de conhecimento, possibilitando uma abordagem pedagógica mais consistente e que alcance resultados mais satisfatórios [Bano et al. 2018]. Para apoiar a construção de um arcabouço tecnológico que auxilie professores e estudantes a construírem uma interação mais efetiva, pesquisadores têm proposto diversas abordagem para construção de sistemas que se fundamentam nos conceitos de Sistemas Adaptativos e Inteligentes para Educação (SAIEs). Esse tipo de sistema é capaz de alterar suas características para atender às necessidades dos usuários. Esses ambientes analisam características importantes do estudante e fazem ajustes apropriados ao ambiente para auxiliar e aprimorar a aprendizagem [Durlach and Lesgold 2012]. 
VIII Congresso Brasileiro de Informática na Educação (CBIE 2019)

Anais do XXX Simpósio Brasileiro de Informática na Educação (SBIE 2019)

Seu intuito é criar um ambiente sólido e flexível que auxilie na melhoria de desempenho e no aprendizado dos estudantes.

Muitos SAIEs são construídos de forma híbrida, ou seja, integrados com outros ambientes educacionais com o objetivo de se utilizarem dos dados gerados em conjunto para melhorar a eficiência na adaptação dos conteúdos educacionais [Ferreira et al. 2012]. Uma dessas propostas de construção se dá por meio da integração de SAIEs a Ambientes Educacionais Ubíquos (AEUs) [Settle et al. 2011] - espaços físicos equipados com recursos computacionais, tais como lousas eletrônicas, câmeras, microfones, sensores, etc. capazes de incorporar a visão de computação ubíqua [Weiser 1991] à área de educação. Essas plataformas buscam melhorar a interação entre professores, estudantes e o conteúdo didático, com a integração dessas tecnologias ao ambiente de ensino de forma transparente e colaborativa. No entanto, ainda são raras as propostas que utilizam dos dados gerados por essas plataformas para verificar seu real ganho educacional.

Neste sentido, este trabalho apresenta um estudo que visa investigar a efetividade da utilização de SAIEs integrados a AEUs. O objetivo é verificar se existe diferença estatística no aprendizado dos estudantes para diferentes perfis de utilização, ou seja, verificar se o nível de utilização da plataforma influencia no desempenho final do estudante. Para conduzir o estudo, duas perguntas de pesquisa foram definidas: (P1) O desempenho do estudante está associado ao seu nível de comportamento em um AEU? (P2) Quais os perfis de acesso apresentam melhores e piores desempenhos quando associados à utilização de um AEU? Essas questões foram exploradas utilizando: (a) revisão da literatura; (b) análise de comportamento e interações dos estudantes; (c) processo de agrupamento de dados (clusterização); e (d) análises estatísticas.

O experimento contou com a participação de 119 estudantes, divididos em seis turmas da disciplina de Interação Humano-Computador e Arquitetura e Organização de Computadores ao longo de três semestres (2016/2, 2017/1 e 2017/2). Um algoritmo de agrupamento foi utilizado para ajudar a definir diferentes grupos sob a perspectiva de como utilizam o ambiente, isto é, seu perfil de interação. Algoritmos de agrupamento são interessantes para esse tipo de pesquisa pois visam agrupar instâncias (estudantes) com características semelhantes em um mesmo grupo, permitindo assim, a identificação de perfis estratégicos para uma análise mais consistente e confiável [Hair et al. 2009]. Sob a perspectiva estatística, o teste de Kruskal-Wallis foi utilizado para verificar se existem diferenças significativas entre os grupos analisados. Os resultados deste estudo podem auxiliar tanto professores quanto estudantes. Os primeiros, ao decidirem sobre a utilização de uma plataforma ubíqua para auxiliar no processo de ensino presencial, bem como, ter uma noção dos perfis de comportamento que são mais propícios a ter resultados positivos quanto utilizarem a plataforma. Os segundos, a descobrir como seria a melhor forma para se comportar buscando um melhor rendimento durante seus estudos.

O restante do artigo está estruturado da seguinte maneira: na Seção 2, é apresentado o estado da arte acerca do tema central da pesquisa; na Seção 3, são detalhados os métodos e procedimentos para realização dos experimentos; na Seção 4, são descritos e discutidos os resultados estatísticos obtidos; e, por fim, na Seção 5, são feitas as considerações finais e sugeridos trabalhos futuros. 
VIII Congresso Brasileiro de Informática na Educação (CBIE 2019)

Anais do XXX Simpósio Brasileiro de Informática na Educação (SBIE 2019)

\section{Estado da Arte}

Diferentes técnicas computacionais têm surgido com o intuito de auxiliar neste processo de análise de dados sobre as características dos estudantes. Uma que vem ganhando destaque nas últimas décadas é a Learning Analytics [Siemens and Baker 2012]. Definida como a medição, coleta e análise de dados sobre os estudantes em ambientes educacionais, possui o objetivo de realizar uma interpretação individualizada do percurso, das dificuldades e das características de estudo dos estudantes [Siemens and Baker 2012].

Uma importante faceta que explora essa técnica foi introduzida por [Shum e Crick 2012] e é conhecida como Dispositional Learning Analytics. Essa estratégia combina os dados gerais de aprendizagem com elementos "disposicionais" próprios dos estudantes, incluindo seus comportamentos, suas atitudes e seus valores [Tempelaar et al. 2018]. A Dispositional Learning Analytics vai além da análise de dados sobre o estudante, pois combina também facetas comportamentais e cognitivas dos indivíduos [Rienties et al. 2017]. Como parte importante desta proposta, este artigo utiliza mecanismos da Dispositional Learning Analytics com o intuito de verificar se estudantes com interações diferentes também apresentam desempenho diferente.

Diversos algoritmos têm sido empregados para auxiliar no processo de modelagem comportamental do estudante. Alguns deles se caracterizam por utilizar abordagens fundamentadas em ontologias, permitindo a definição de um modelo extensível, reutilizável e adaptável a diferentes domínios [Júnior et al. 2017, Júnior e Dorça 2018, Costa et al. 2018]. Outras abordagens se fundamentam na utilização de Redes Neurais ou modelos genéticos que permitem a construção de modelos mais adaptáveis às características reais dos estudantes [Severo and Freitas 2018, Oliveira et al. 2018]. Também é possível encontrar modelos que se baseiam em técnicas de mineração de dados para realizar a classificação, predição ou agrupamento das informações [Harris and Kumar 2018, De Los Reyes et al. 2019]. Um diferencial importante deste trabalho refere-se a sua capacidade de utilizar um mecanismo da mineração de dados (algoritmos de agrupamento) aplicado a estudantes matriculados em cursos presenciais com o intuito de estabelecer uma diretriz baseada no comportamento. A proposta analisa se estudantes com diferentes perfis de comportamento também apresentam diferença significativa no desempenho. Raras são as pesquisas disponíveis na literatura que abordam tal análise.

\section{Materiais e Métodos}

Esta seção descreve os materiais e métodos aplicados no planejamento para realização do experimento proposto para este estudo.

\subsection{Participantes}

Participaram deste estudo 119 estudantes distribuídos em seis turmas de três semestres (2016/2, 2017/1, 2017/2). Foram utilizadas três turmas da disciplina Interação HumanoComputador (IHC) e três turmas da disciplina Arquitetura e Organização de Computadores (AOC), todas ministradas de forma presencial para o ensino superior na Universidade Federal de Uberlândia (UFU). Durante os três semestres em que o estudo foi realizado, os professores das disciplinas apresentaram aos estudantes uma nova ferramenta, baseada nos conceitos de SAIEs e de AEUs, para auxiliá-los nos estudos. Após uma demonstração inicial da plataforma no início de cada disciplina, as aulas passaram a ser ministradas 
VIII Congresso Brasileiro de Informática na Educação (CBIE 2019)

Anais do XXX Simpósio Brasileiro de Informática na Educação (SBIE 2019)

utilizando-se a plataforma, sem alterar a dinâmica de trabalho e didática do professor. As informações dadas em aula ficavam então posteriormente disponíveis para acesso na plataforma.

\subsection{Questões de Pesquisa}

O principal objetivo dessa pesquisa é verificar se a utilização de um AEU como ferramenta para apoio ao ensino presencial pode contribuir para a melhora na aprendizagem dos estudantes. Como pontos específicos, será verificado se diferentes comportamentos ao utilizarem a plataforma ubíqua correspondem a diferentes níveis de aprendizagem. Dessa forma, é possível definir duas questões de pesquisa que irão guiar este estudo:

a) O desempenho do estudante pode está associado ao seu nível de comportamento em um AEU?

b) Quais os perfis de acesso apresentam melhores e piores desempenhos quando associados a utilização de um AEU?

\subsection{Procedimentos}

O experimento foi realizado de forma a não alterar a maneira como o professor ministrava suas aulas. No início do semestre, os estudantes foram orientados sobre como utilizar a plataforma. Tal utilização era opcional, servindo apenas como um complemento para os estudos. Os procedimentos para realização do experimento podem ser divididos em três fases: aquisição dos dados, quantificação das interações e agrupamento dos dados.

\subsubsection{Fase 1: Aquisição dos Dados}

Durante as aulas, todo o conteúdo apresentado pelo professor era capturado e armazenado pela plataforma ubíqua, permitindo assim o acesso futuro ao conteúdo. Com esse recurso, os estudantes poderiam focar sua atenção no conteúdo sendo ministrado pelo professor, com a certeza de que todas as informações estariam disponíveis posteriormente para acesso. O conteúdo capturado pelo sistema era automaticamente segmentado e transformado em Objetos de Aprendizagem (OAs). Foram utilizados um total de 110 aulas e 5220 OAs.

Durante o semestre letivo, os estudantes utilizavam a plataforma para estudos regulares. É importante ressaltar que todas as interações dos estudantes com o material educacional estavam sendo registradas pela plataforma educacional (logging). Com isso, era possível saber como foi o comportamento de cada estudante e quais atividades eram realizadas durante seus estudos. Esse registro ocorreu ao longo de todos os semestres letivos e as interações foram capturadas e armazenadas na forma de $\log s$ de acesso.

\subsubsection{Fase 2: Quantificação das interações}

Para se ter uma medida mais quantitativa sobre o acesso dos estudantes, foi realizado um estudo com o intuito de definir limites para o tempo de acesso a cada material educacional. Dessa forma, foi possível definir três níveis de tempo para as interações (interações curtas - intA; interações médias - intB; e interações longas - intC), associados a cada parte 
VIII Congresso Brasileiro de Informática na Educação (CBIE 2019)

Anais do XXX Simpósio Brasileiro de Informática na Educação (SBIE 2019)

do material. Após a definição desses limiares, foram executados procedimentos para quantificar tais interações, permitindo assim uma visão global de como foram os acessos feitos pelos estudantes em cada turma analisada. A Tabela 1 apresenta a quantidade de interações em cada categoria analisada para cada umas das turmas.

Tabela 1. Total de interações por turma analisada
\begin{tabular}{c|cccc}
\hline Turma & intA & intB & intC & Total \\
\hline \hline 1 & 12802 & 6131 & 41982 & 60915 \\
2 & 5973 & 2823 & 21988 & 30784 \\
3 & 4925 & 2450 & 15625 & 23000 \\
4 & 8025 & 4435 & 29443 & 41903 \\
5 & 8152 & 3792 & 26257 & 38201 \\
6 & 3802 & 1674 & 16711 & 22187 \\
\hline
\end{tabular}

Depois da quantificação das interações, foram realizados procedimentos para verificar e associar os acessos a seus respectivos usuários. Para isso, cada sessão de login foi analisada e foi verificada qual a proporção de interações curtas, médias e longas que cada estudante realizou durante cada acesso. Além disso, foi verificada a sequência de estudo, para tentar identificar estudantes que sempre seguiam um comportamento linear ou estudantes que eram mais interativos. Todas essas informações foram agrupadas por usuário, o que permitiu ter uma medida precisa do nível de comportamento para cada estudante. Neste caso, definiu-se uma estrutura para representação de um conjunto de interações:

$$
I S=\{U s, p A(\%), p B(\%), p C(\%)\}
$$

Onde:

$I S=$ Conjunto de interação;

Us = Usuário;

$p A=$ Proporcionalidade de interações curtas;

$p B=$ proporcionalidade de interações médias;

$p C=$ proporcionalidade de interações longas .

\subsubsection{Fase 3: Abordagem de Agrupamento de Dados}

Após a quantificação das interações por usuário, foi realizado um procedimento para agrupamento de dados (clustering) com o objetivo de agrupar os estudantes com características semelhantes em um mesmo grupo. Em geral, a técnica de agrupamento classifica entidades de modo que cada objeto é semelhante aos outros no agrupamento com base em um conjunto de características (no caso desse estudo, os diferentes níveis de interação). Os grupos resultantes devem apresentar elevada homogeneidade interna (dentro dos agrupamentos) e elevada heterogeneidade externa (entre grupos) [Hair et al. 2009]. O agrupamento foi realizado por meio do algoritmo K-Means, um dos mais objetivos e populares algoritmos de agrupamento disponíveis na literatura [Jain 2010]. Seu princípio é encontrar $\mathrm{K}$ grupos nos dados fornecidos. $\mathrm{O}$ algoritmo funciona iterativamente para atribuir 
VIII Congresso Brasileiro de Informática na Educação (CBIE 2019)

Anais do XXX Simpósio Brasileiro de Informática na Educação (SBIE 2019)

cada instância a um dos grupos $\mathrm{K}$ com base nos recursos fornecidos. As instâncias são agrupadas com base na similaridade das características.

A Tabela 2 apresenta os dados centrais obtidos a partir do processo de agrupamento. É possível perceber que o cluster2 (CL2) possui maiores valores para $p A(33.17)$ e $p B(19.73)$ e por sua vez, menores valores para $p C$ (46.67). Já o cluster1 (CL1) possui os maiores valores de $p C$ (79.37) e menores de $p A$ (15.18) e $p B(5.06)$. Por fim, o cluster0 $(C L O)$ possui valores medianos para $p A$ (24.03), $p B$ (11.23) e $p C$ (64.46).

Tabela 2. Distribuição média das interações em cada cluster.

\begin{tabular}{c|ccc}
\hline \multirow{2}{*}{ Atributo } & \multicolumn{3}{|c}{ Clustering } \\
& $C L O(\%)$ & $C L 1(\%)$ & $C L 2(\%)$ \\
\hline \hline$p A$ & 24.03 & 15.18 & 33.17 \\
$p B$ & 11.23 & 5.06 & 19.73 \\
$p C$ & 64.46 & 79.37 & 46.67 \\
\hline
\end{tabular}

\section{Resultados e Análises}

Após o processo de agrupamento, foram executados testes estatísticos para verificar se existe ou não diferenças significativas no desempenho dos estudantes em cada cluster: (CLO com 49 estudantes, CL1 com 52 estudantes e, por fim, CL3 com 18 estudantes). A Figura 1 apresenta os gráficos de dispersão desta estratégia no qual é possível ver a distribuição dos estudantes em cada grupo. Na Figura 1(a), o eixo X representa valores para $p C$ e o eixo Y representa valores de $p B$. Já na Figura 1(b), o eixo X representa valores para $p C$ e o eixo Y representa valores de $p A$.

Figura 1. Gráficos de dispersão para a distribuição dos clusters.

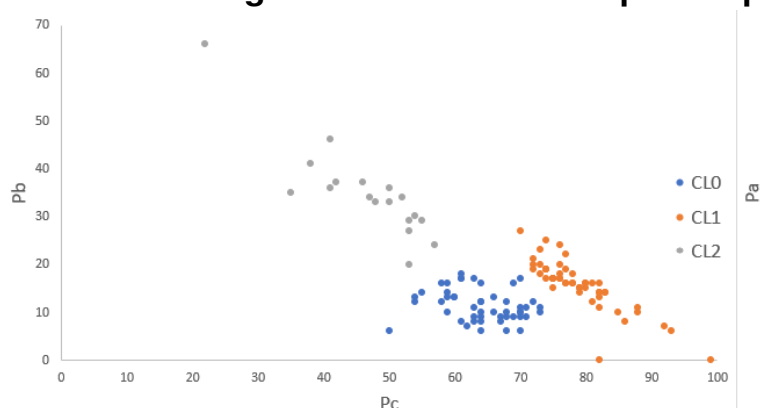

(a)

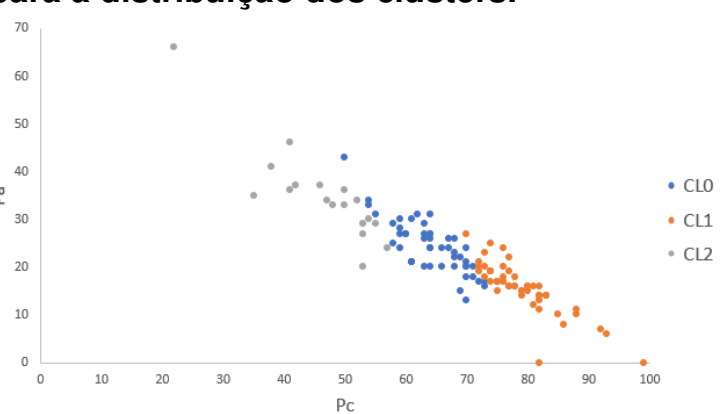

(b)

Para verificar se as notas dos estudantes apresentaram alguma diferença entre os grupos, inicialmente foram realizados dois testes para analisar a homogeneidade de variância e a normalidade das amostras. Para a homogeneidade, foi utilizado o teste de Leveni. Para verificar se os resíduos das amostras seguem a distribuição normal, foi utilizado o teste de Shapiro-Wilk. Com os testes, foi possível concluir que as amostras são homogêneas ( $p$-value $=0,118)$. No entanto, o teste de Shapiro-Wilk mostrou que as amostras analisadas não apresentaram normalidade residual $(\mathrm{W}(p)=0,908(0,00))$.

Esses resultados orientaram a escolha do próximo teste para verificar a diferença entre as médias: o teste não paramétricos Kruskal-Wallis, que mostrou que o comportamento dos estudantes interfere no seu desempenho $(H(2)=7,063 ; p<0,05)$. Neste caso, 
VIII Congresso Brasileiro de Informática na Educação (CBIE 2019)

Anais do XXX Simpósio Brasileiro de Informática na Educação (SBIE 2019)

foi possível identificar que existe uma diferença estatisticamente significativa entre as notas dos estudantes que estão agrupados nos diferentes clusters. Na Tabela 3 é possível observar que os estudantes do $C L 1$ possuem as maiores médias de rank e os estudantes do CL2 possuem as menores. Tabela 3. Médias de desempenhos e resultado do teste de Kruskal-Wallis em
cada cluster.

\begin{tabular}{c|cccc} 
Cluster & $\mathrm{N}^{1}$ & Mean Rank & Chi-square $(\mathrm{p})^{2}$ & $\mathrm{df}$ \\
\hline $\mathrm{Cl0}$ & 49 & 60,74 & & \\
$\mathrm{Cl} 1$ & 52 & 65,90 & $1103,00(0,032)$ & 2 \\
$\mathrm{Cl} 2$ & 18 & 40,92 & & \\
\hline
\end{tabular}

${ }^{1}$ Número de estudantes no cluster. ${ }^{2} p$-value $<0,05$ indica que existe diferença significativa entre as médias dos grupos analisado.

Os estudantes classificados no $C L 1$ tiveram as maiores notas. Esse grupo é justamente o grupo que possui acessos mais longos $(p C)$ e poucos acessos curtos $(p A)$. Já os estudantes classificados no $C L 2$ são os estudantes com menores notas. Esses estudantes possuem o perfil mais mediano, aqueles que apresentam comportamento não tão longo nem tão curto (Tabela 2). Os estudantes classificados no CLO apresentam comportamento mais dinâmico, fazendo o uso do ambiente menos frequente e com muitos acessos rápidos.

Também é importante identificar onde realmente está a diferença estatisticamente significante. Para isso, foi utilizada a abordagem de Dunn-Bonferroni para realização da comparação par a par (pairwise comparisons). Na Tabela 4, é possível verificar a diferença que existe entre os três grupos. Há uma diferença significante entre o CL2 e o $c L 1(p=0,024)$. Neste caso, o grupo que possui maior média (estudantes que acessam o ambiente prioritariamente por longos períodos) e o grupo que possui menor média (estudantes que costumam não acessar o sistema por longos períodos). O gráfico de Bloxplot na Figura 2 apresenta de forma mais clara a visualização dessas diferenças entre as médias. É possível perceber que a diferença entre $C L O$ e $C L 1$ e entre $C L O$ e $C L 2$ não foi tão expressiva quanto a diferença entre $C L 1$ e $C L 2$.

Tabela 4. Resultado de teste de Comparação par a par entre os clusters.

\begin{tabular}{c|ccccc} 
Cluster & Teste Estatístico & Erro padrão & Padrão de Teste & Sig & Ajuste de Sig $^{1}$ \\
\hline Cl2 - C10 & 19,82 & 9,50 & 2,08 & .037 & .111 \\
Cl2 - Cl1 & 24,98 & 9,42 & 2,65 & .008 & $\mathbf{. 0 2 4}$ \\
C10 - C11 & $-5,15$ & 6,86 & -.752 & .452 & 1.00 \\
\multicolumn{4}{r}{${ }^{1}$ Valor de ajuste $<0,05$ indica diferença significativa entre os grupos analisados. }
\end{tabular}

Analisando o comportamento dos quartis e das medianas representadas por cada cluster, é possível perceber uma maior influência da variável que representa o acesso longo $(p C)$ no resultado final do desempenho dos estudante. Essa é a variável que mais influenciou no desempenho de cada cluster, ou seja, quanto maior é a ocorrência de $p C$ maior é a média no desempenho no cluster; e quanto menor é a ocorrência de $p C$, menor é a média no desempenho. Existe também uma influência da variáveis de acessos curtos $(p A)$, não tão significativa quanto $p C$, mas capaz de representar uma certa influência sobre os clusters. No caso da variável $p A$, quanto menor são os acessos curtos, maiores são as médias no desempenhos dos estudantes. Com isso, é possível perceber que a variável 
Figura 2. Comparação entre os três clusters.

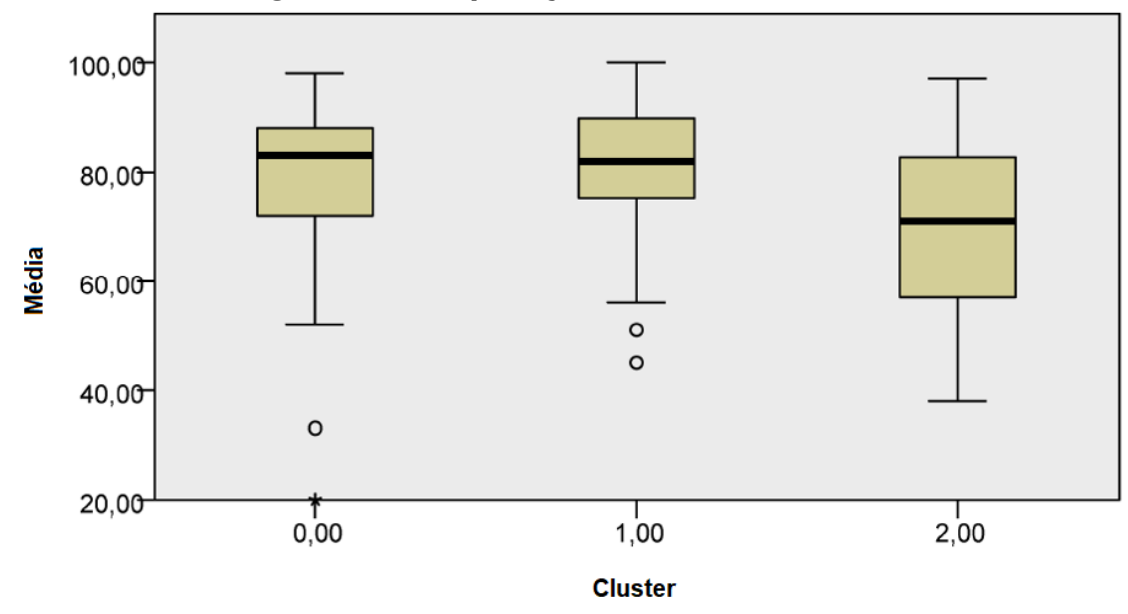

$p C$ influencia positivamente no desempenho do estudante ao passo que a variável $p A$ influencia, de certa forma, negativamente no desempenho do estudante.

Respondendo a primeira questão de pesquisa proposta, foi possível provar estatisticamente que existe sim relação entre o comportamento dos estudantes durante a utilização de um AEU e o seu desempenho. O teste de Kruskal-Wallis mostrou um maior desempenho no mesmo grupo de estudantes (os que acessam por períodos mais longos) e um menor desempenho para aqueles que não acessam por períodos longos.

Respondendo a segunda questão de pesquisa proposta, é possível dividir o grupo de estudantes que fez uso da plataforma em três perfis: o Perfil A são os estudantes que possuem mais acessos longos e menor acesso curto e médio; o Perfil $B$ são os estudantes que acessam o sistema com menos acessos longos e mais acessos curtos e médios; e o Perfil $C$ são os estudantes que possuem um comportamento mais mediano, estando na média para acessos curtos, médios e longos. É possível concluir, estatisticamente, que os estudantes do Perfil A possuem as melhores notas, ao passo que os estudantes do Perfil $B$ possuem as menores. Os estudantes do Perfil $C$ se enquadram em uma região de mais incerteza, na qual não é possível fazer afirmações com fortes fundamentos estatísticos.

\section{Conclusão}

Este artigo explorou a integração de uma plataforma híbrida, fundamentada em conceitos de SAIEs e AEUs, para verificar se o nível de interações com o sistema pode influenciar no desempenho dos estudantes. O objetivo principal foi verificar se existe diferença estatística no aprendizado dos estudantes para diferentes perfis de comportamento. Nos experimentos, foram acompanhadas seis turmas durante três semestres letivos, englobando um total de 119 estudantes. Para todas as turmas, foi utilizada uma plataforma de ensino ubíqua que faz o uso de ferramentas para monitoramento do comportamento, com o intuito de auxiliar professores e estudantes em suas atividades. Foram coletadas interações dos estudantes com a plataforma ubíqua, permitindo identificar o comportamento de cada estudante durantes seus estudos. Um algoritmo de agrupamento foi utilizado para diferenciar o perfil dos estudantes com base em características relacionadas ao tempo de interação em cada parte do material didático. Após tal procedimento, os grupos resultantes foram associados com suas respectivas notas finais na disciplina e testes estatísticos 
VIII Congresso Brasileiro de Informática na Educação (CBIE 2019)

Anais do XXX Simpósio Brasileiro de Informática na Educação (SBIE 2019)

foram executados para verificar se existiam diferenças significativas entre as médias das notas para os estudantes classificados em cada grupo.

Os resultados mostraram que o nível de interação com o ambiente, ou seja, o comportamento do estudante, tem influência direta (comprovada estatisticamente) no desempenho do estudante. Para todos os grupos analisados, os estudantes que permanecem mais tempo no ambiente educacional possuem melhores desempenhos na disciplina do que os estudantes que permanecem menos tempo. Por outro lado, os estudantes que acessam a plataforma educacional prioritariamente por curtos períodos tendem a apresentarem menores notas no final da disciplina. Com isso, é possível concluir que a partir das interações dos estudantes é possível classificá-los em determinados perfis, e estes perfis podem ajudar a determinar o nível de desempenho do estudante.

Com tudo o que foi discutido até aqui, esse estudo recomenda a utilização de plataformas de ensino ubíquas como ferramenta de apoio ao ensino presencial, com potencial de contribuir positivamente para a melhoria no ensino e auxiliar os professores a identificar perfis de estudantes suscetíveis a sucesso ou fracasso na disciplina, com base no seu comportamento. Um Modelo de Estudante pode auxiliar a plataforma ubíqua a criar mecanismos para rastrear as interações dos estudantes e, com isso, melhorar a dinâmica de apresentação e recomendação de conteúdo. Ainda, esse trabalho também recomenda que o processo de agrupamento por meio da plataforma ubíqua pode trazer resultados positivos ao ensino, pois, além de facilitar a visualização de diferentes características internas dos grupos, permite que ações pedagógicas possam ser tomadas individualmente para cada grupo, buscando com isso, a melhoria na qualidade do ensino.

\section{Agradecimentos}

Os autores agradecem o apoio de CNPq, FAPEMIG, PROPP/UFU, FACOM/PPGCO/PROAP/UFU, IFSULDEMINAS e PET/MEC/SESu. O presente trabalho foi realizado com apoio da Coordenação de Aperfeiçoamento de Pessoal de Nível Superior - Brasil (CAPES) - Código de Financiamento 001.

\section{Referências}

Bano, M., Zowghi, D., Kearney, M., Schuck, S., e Aubusson, P. (2018). Mobile learning for science and mathematics school education: A systematic review of empirical evidence. Computers \& Education, 121:30-58.

Costa, L., Sanches, L., Nascimento, L., Souza, M., e Amorim, R. J. R. (2018). Ontolo: Um modelo ontológico para avaliar o desempenho acadêmico na educação a distância. In Simpósio Brasileiro de Informática na Educação (SBIE 2018), p. 1898-1902.

De Los Reyes, D. A. G., Thomas, E. A., da Rosa, L. L., e Neto, W. P. G. (2019). Student success prediction: An analysis of the demand for a transfer learning approach. Revista Brasileira de Informática na Educação (RBIE), 27(01):01.

Durlach, P. J. e Lesgold, A. M. (2012). Adaptive technologies for training and education. Cambridge University Press.

Ferreira, H. N. M., Araújo, R. D., de Amo, S., e Cattelan, R. G. (2012). Classroom Experience: A Platform for Multimedia Capture and Access in Instrumented Educational Environments. In Simpósio Brasileiro de Sistemas Colaborativos (SBSC 2012), p. 59-64. 
VIII Congresso Brasileiro de Informática na Educação (CBIE 2019)

Anais do XXX Simpósio Brasileiro de Informática na Educação (SBIE 2019)

Hair, J., Anderson, R., e Babin, B. (2009). Multivariate Data Analysis. Prentice Hall, 7th edition.

Harris, S. C. e Kumar, V. (2018). Identifying student difficulty in a digital learning environment. In IEEE International Conference on Advanced Learning Technologies (ICALT 2018), p. 199-201.

Jain, A. K. (2010). Data clustering: 50 years beyond k-means. Pattern Recognition Letters, 31(8):651-666.

Júnior, C. B. and Dorça, F. (2018). Uma abordagem para a criação e recomendação de objetos de aprendizagem usando um algoritmo genético, tecnologias da web semântica e uma ontologia. In Simpósio Brasileiro de Informática na Educação (SBIE 2018), p. $1533-1542$.

Júnior, C. P., Francisco, R., Silva, L., Veiga, E., Fernandes, M., e Dorça, F. (2017). Uso de ontologias para agentes conversacionais no contexto de ensino-aprendizagem: Uma revisão sistemática da literatura. In Simpósio Brasileiro de Informática na Educação (SBIE 2017), p. 183-192.

Oliveira, H. L., Vivas, A., Assis, L., Pitangui, C., de Almeida, A. M. F., e Dorça, F. (2018). Detecção automática e dinâmica de estilos de aprendizagem para sistemas adaptativos inteligentes para a educação utilizando a meta-heurística vitis vinífera. In Simpósio Brasileiro de Informática na Educação (SBIE 2018), p. 1323-1332.

Rienties, B., Cross, S., e Zdrahal, Z. (2017). Implementing a learning analytics intervention and evaluation framework: What works? In Big data and learning analytics in Higher Education, p. 147-166. Springer.

Settle, A., Dettori, L., e Davidson, M. J. (2011). Does Lecture Capture Make a Difference for Students in Traditional Classrooms. In Annual Joint Conference on Innovation and Technology in Computer Science Education (ITiCSE' 17), pages 78-82.

Severo, C. E. e Freitas, R. (2018). Socialsearch: uma ferramenta de apoio pedagógico para mediação da aprendizagem nas redes sociais digitais. In Simpósio Brasileiro de Informática na Educação (SBIE 2018), p. 1583-1592.

Shum, S. B. e Crick, R. D. (2012). Learning dispositions and transferable competencies: pedagogy, modelling and learning analytics. In International Conference on Learning Analytics and Knowledge (LAK' 12), p. 92-101.

Siemens, G. e Baker, R. S. J. d. (2012). Learning analytics and educational data mining: Towards communication and collaboration. In International Conference on Learning Analytics and Knowledge (LAK' 12), p. 252-254.

Tempelaar, D., Rienties, B., Mittelmeier, J., e Nguyen, Q. (2018). Student profiling in a dispositional learning analytics application using formative assessment. Computers in Human Behavior, 78:408-420.

Weiser, M. (1991). The computer for the 21st century. Scientific American, 265(3):94104. 